\title{
STAR CLUSTER SIMULATIONS ON HARP
}

\author{
SVERRE J. AARSETH \\ Institute of Astronomy, University of Cambridge, UK
}

\begin{abstract}
We describe some aspects of implementing star cluster simulations on HARP. The code NBODY4 employs the Hermite scheme with hierarchical block-steps for direct integration. The algorithms have been optimized for parallel processing with the eight pipeline HARP-2 delivering a peak performance of about 1.7 Gflops for $N=10^{4}$ particles. Hard binaries are studied by $\mathrm{KS}$ regularization which also uses the Hermite scheme, whereas strong interactions between $3-5$ particles are treated by chain regularization. Astrophysical processes modelled include mass loss by stellar evolution, two-body tidal interaction, Roche lobe mass transfer, common envelope evolution, magnetic braking and gravitational radiation. Consistent values of stellar radii and evolution type are obtained by fast look-up. A new formulation of collision outcomes yields blue stragglers and other exotic objects. Some recent results for an open cluster model are presented.
\end{abstract}

\section{Introduction}

The advent of the HARP special-purpose computer represents a watershed in the history $N$-body simulations. In the present paper, we describe a new star cluster project undertaken with the eight pipe-line HARP-2 version which was installed at the Institute of Astronomy in August 1994. Until now, it has been feasible to perform such calculations with up to $N=2500$ members on dedicated workstations, with just one model requiring several months of continuous work (Heggie and Aarseth 1992). Only by the most heroic effort on a supercomputer (i.e. more than $1000 \mathrm{CPU}$ hours over two years) has it been possible to reach the magical limit $N=10^{4}$ (Spurzem and Aarseth 1996). Using HARP-2, we can now investigate a range of different parameters for star cluster models with (say) $N=10^{4}$. In order to reach this objective, it has been necessary to develop software for all the relevant 
processes occurring in star clusters and to adapt the code in a form suitable for HARP. The main processes which have been implemented for studying open clusters can be summarized as follows:

- Galactic tidal field

- Primordial binaries

- Mass loss from evolving stars

- Velocity kick during neutron star formation

- Tidal circularization

- Roche lobe mass transfer

- Common envelope evolution

- Magnetic braking

- Gravitational radiation

- Physical stellar collisions

- Formation of exotic objects.

In the subsequent sections we discuss briefly the astrophysical processes listed above. However, we begin by describing the main technical methods concerned with the actual integration since these aspects have required much new work for applications on HARP.

\section{Hermite Integration Scheme}

The basic integration scheme for HARP computers exploits the fast evaluation of the total force (per unit mass) and its first time derivative by employing a fourth-order formulation (Makino 1991). We write a Taylor series for each of these quantities as

$$
\begin{aligned}
& \mathbf{F}=\mathbf{F}_{0}+\dot{\mathbf{F}}_{0} t+\frac{1}{2} \ddot{\mathbf{F}}_{0} t^{2}+\frac{1}{6} \dddot{\mathbf{F}}_{0} t^{3} \\
& \dot{\mathbf{F}}=\dot{\mathbf{F}}_{0}+\ddot{\mathbf{F}}_{0} t+\frac{1}{2} \dddot{\mathbf{F}}_{0} t^{2} .
\end{aligned}
$$

These equations can be inverted to obtain the second and third time derivatives in terms of $\mathbf{F}$ and $\dot{\mathbf{F}}$ at the begining and end of a time-step, which can then be used to correct the coordinates and velocities.

The so-called Hermite integration scheme yields increased efficiency by the introduction of hierarchical time-steps $\Delta t_{n}=1 / 2^{n-1}$, combined with a hardware chip for fast prediction of all coordinates and velocities to order $\dot{\mathbf{F}}$. Depending on the natural time-step obtained from some suitable convergence criterion, the new step may then be reduced by 2 (or even 4) or remains the same each time, but can only be increased by 2 every other step to maintain time commensurability. We note that this hierarchical treatment achieves a performance which is weakly dependent on the time-step 
range (i.e. depth of the potential well) for the same particle number. Moreover, a given fraction of the peak performance is reached at larger values of $N$ when the number of pipe-lines is increased to match the increased number of particles in the same block level.

Close binaries and hyperbolic fly-bys are integrated by KustaanheimoStiefel (1964, hereafter KS) regularization. The equations of motion are

$$
\begin{aligned}
\mathbf{u}^{\prime \prime} & =\frac{1}{2} h \mathbf{u}+\frac{1}{2} R \mathcal{L}^{T} \mathbf{P} \\
h^{\prime} & =2 \mathbf{u}^{\prime} \cdot \mathcal{L}^{T} \mathbf{P} \\
t^{\prime} & =\mathbf{u} \cdot \mathbf{u}
\end{aligned}
$$

Here $\mathbf{u}$ denotes the new coordinates which satisfy the relation $\mathbf{u} \cdot \mathbf{u}=R, h$ is the two-body binding energy per unit mass, $\mathcal{L}(\mathbf{u})$ is a $4 \times 3$ matrix and $\mathbf{P}$ is the physical tidal perturbation exerted by the other particles. Decisionmaking for existing KS solutions is based on the relative perturbation, $\gamma=P R^{2} / m_{b}$, with $R$ the separation and $m_{b}$ the combined mass of the components. Further discussions together with the relevant transformations can be found elsewhere (Aarseth 1985, 1994).

In order to achieve uniformity, we replace the standard difference scheme (Aarseth 1985) by a Hermite KS formulation. This entails differentiating Eqns. (3) and (4), making use of the linear matrix property $\mathcal{L}^{\prime}(\mathbf{u})=\mathcal{L}\left(\mathbf{u}^{\prime}\right)$, whereas the time transformation (5) yields $\mathbf{P}^{\prime}=R \dot{\mathbf{P}}$. Although the new equations of motion contain more terms and also require velocity predictions of perturbers as well as a KS velocity transformation for evaluating $\dot{\mathbf{P}}$, the additional effort is to some extent compensated by a simpler treatment.

All the calculations relating to KS are carried out on the host computer and may therefore present an unbalanced load when integrating many perturbed binaries. This problem is alleviated by employing a slow-down scheme (Mikkola and Aarseth 1996) for weakly perturbed binaries (say $\gamma<5 \times 10^{-5}$ ). The main idea is to exploit the adiabatic invariance such that one KS orbit with augmented perturbation may represent several physical orbits, while maintaining a correct treatment of the secular effects. As before, binaries with small relative perturbations $\left(\gamma<10^{-6}\right)$ are assumed to be unperturbed and advanced one or more periods without any stepwise integration. Moreover, hierarchical triples are treated as composite KS binaries while satisfying a criterion of dynamical stability (Eggleton and Kiseleva 1995). Finally, we remark that an energy stabilization term is included in Eqn. (3) which introduces a small systematic error in the eccentricity. However, this effect is reduced considerably when using the slow-down scheme. 


\section{Close Encounters}

The treatment of close encounters on HARP requires many new considerations. Here we summarize some of the technical problems which must be overcome. Since the fictitious time defined by Eqn. (5) is not a linear function of time, each KS solution is advanced one or more steps within a new block-step before integrating the single particles and corresponding centre-of-mass (c.m.) particles.

We distinguish between active and inactive c.m. particles, where the latter are associated with unperturbed KS solutions; hence their new force can be evaluated as for single particles. To obtain a consistent force on an active c.m. particle, we include the contributions from distant members in one summation and add the mass-weighted force on the two components due to the perturbers. The case of several active c.m. particles in the same block can then be treated by obtaining the force on HARP for each one separately after defining a mask (i.e. by specifying zero masses) for the relevant perturbers. However, in order to utilize more than one pipe-line simultaneously, we first form a joint perturber list which acts as a mask for the different c.m. forces on HARP, and then add all these perturber contributions on the host. Although this algorithm requires a careful sifting to avoid multiplicity, the additional effort on the host is minimized by taking into account the spacing between different c.m. particles and their corresponding maximum perturber distances which can be estimated from the selection criteria based on binding energy and mass ratio.

The strategy for determining KS candidates exploits the property that such particles have small time-steps and occupy the deepest block-step levels. Hence it is only necessary to form a candidate list when the current level has few members, thereby reducing the effort. Once two particles have been selected for KS treatment, the initialization proceeds as before (Aarseth 1994). However, the commensurability requirement sometimes leads to unduly small values of the c.m. time-step with loss of efficiency in the case of short-lived interactions (i.e. wide binaries).

It is desirable to delay the termination of a KS treatment until the end of a block-step. This entails advancing the regularized solution some fraction of the current block-step, where the latter is likely to be quite small; say, the corresponding c.m. time-step in the case of a strong interaction. Now the loss of efficiency is less noticeable since the new steps for direct integration are usually small.

Several tasks can be carried out while HARP is busy with force evaluations, such as the prediction of the next set of particles (if any) as well as any KS coordinates and velocities, the construction of the joint perturber list and noting particles with small steps. All these tasks are performed 
during the first HARP call, whereas the corrector is included for the previous set of particles on any subsequent calls. The full optimization by socalled low-level functions enhances the performance from about 1.1 Gflops to 1.7 Gflops for $N=10^{4}$, which in practice means twice the throughput of a Cray-YMP supercomputer using the vectorized NBODY5 neighbour scheme code (Spurzem and Aarseth 1996).

Perturbed chain regularization (Mikkola and Aarseth 1993) has also been implemented. Typically one large simulation employs the chain procedures about 100 times; however, most of these multiple encounters of three or four particles involve extremely energetic interactions. For instance, the chain code is used to study binary-binary encounters. Such systems may decay into a compact triple and an escaping body; alternatively a triple or quadrupole may increase the membership by an intruding particle.

Finally, the practical usefulness of obtaining neighbour lists on HARP should also be emphasized. Several strategies have been developed in order to deal with the different requirements, such as finding the nearest neighbour or constructing new perturber lists.

\section{Cluster Model}

We now describe the initial conditions for an open cluster model with $N=10^{4}$ members. The coordinates and velocities are generated from an equilibrium King model with central concentration $W_{0}=7$ in an exterinal tidal field (Heggie and Ramamani 1992). We choose a modern IMF (Kroupa, Tout and Gilmore 1993) with masses in the range $10-0.2 m_{\odot}$. The corresponding tidal radius in the solar neighbourhood is then $R_{t}=22$ pc, with half-mass radius $R_{h} \simeq 3 \mathrm{pc}\left(r_{h}=0.82\right.$ in scaled units) and rms velocity $V_{0} \simeq 2 \mathrm{~km} / \mathrm{s}$, giving a crossing time of $\simeq 4 \times 10^{6} \mathrm{yr}$.

The main aim of the present project is to study open clusters containing a significant proportion of primordial binaries. Although the dynamical effects of such binaries have been investigated before (McMillan, Hut and Makino 1990, 1991, Heggie and Aarseth 1992), the emphasis here is to include all relevant astrophysical processes which are outlined in the subsequent sections (but also see Aarseth 1996 for an earlier review). We adopt an initial binary fraction $f_{0}=N_{b} /\left(N_{b}+N_{s}\right)$ of $5 \%$ with $N_{b}=500$ binaries and $N_{s}=9500$ single stars, and a period distribution based on the solar neighbourhood (Duquennoy and Mayor 1991). Here the individual binary components are selected from the same IMF as the single stars but even so their combined masses do not tend to exceed the latter because of sampling effects. First we restrict the uncorrelated period to a minimum value of one day and, for practical reasons, impose an upper cutoff at a semi-major axis of $a_{\max }=48 \mathrm{AU}$ which is somewhat smaller than that of a hard binary. 
According to recent ideas (Kroupa 1995), the closest binaries experience some changes in their two-body elements during the pre-main-sequence evolution. Consequently, we modify the semi-major axis $(a)$ and eccentricity (e) according to tidal circularization theory (Mardling 1995), assuming a time interval of $10^{5} \mathrm{yr}$ and an initial stellar radius of fifteen times the ZAMS value. This procedure yields 25 circularized binaries, and other binaries with some modifications of $a$ and $e$. Hence the eccentricity distribution already contains a small proportion of short-period circular orbits.

\section{Synthetic Stellar Evolution}

The modelling of several astrophysical effects requires knowledge of the stellar mass and radius which change with time. The stars are characterized by an index $K=0,1, \ldots, 10$, according to their temporal location in the HR-diagram; from low-mass main-sequence to neutron stars and even black holes or exotic objects. We adopt a continuous mass loss by stellar winds according to the modified Reimers expression (Tout 1990) beyond the main-sequence $(K>1), \dot{m}=4 \times 10^{-13} R_{*} L / m m_{\odot} / y r$, where the radius $R_{*}$, luminosity $L$ and mass $m$ are in solar units and a suitable time average of $R_{*} L$ is used. We implement mass loss when the accumulated value exceeds one percent. In order to maintain total energy conservation, we include correction procedures for the change in potential energy, together with a modification of the force on neighbouring members or a complete initialization evaluated on HARP, depending on the type of event.

Convenient look-up tables for the evolution of population I stars (Eggleton, Fitchett and Tout 1989) are employed to obtain the stellar radius, luminosity and classification type as a function of the initial mass and age. A solution for the core mass which is used for various procedures has also been introduced (Tout 1995). Each characteristic stage is subdivided into small intervals at which the stellar parameters are updated smoothly.

Neutron star formation is modelled by assuming an asymmetric ejection of the excess mass. The resulting velocity kick is derived from a probability distribution based on recent observations (Lyne and Lorimer 1994). For computational convenience, single stars are restricted to the typical escape velocity, whereas only binary components are assigned the full value in order to test the retention hypothesis. Thus the consistent updating of the stellar parameters permit a synthetic HR-diagram of the remaining cluster members to be constructed as a function of age.

With realistic cluster parameters, physical collisions are inevitable. A general scheme for stellar collision has recently been introduced. We construct a $10 \times 10$ collision matrix for the outcome which depends on stellar type and includes a consistent treatment of common envelope evolution. 


\section{Tidal Circularization}

The growth of stellar radii in close binaries may induce significant tidal dissipation. According to a new treatment for tidal interaction (Mardling 1995), this process may also lead to a chaotic phase of irregular eccentricity behaviour before the final slow approach to a circular orbit. The complete theory has already been implemented in NBODY4 and replaces a previous formulation based on classical tidal capture (Aarseth 1992).

There are two ways in which a tidal interaction may be initiated: (i) increase of eccentricity induced by external perturbations, and (ii) expansion of the stellar radius with time. It has been noted that hierarchical triples may act as catalysts for the former process by producing systematic increase of eccentricity. If a binary is a candidate for tidal interaction (i.e $\left.a(1-e)<10 \max \left(R_{1}, R_{2}\right)\right)$ and the circularization time is below $1 \times 10^{8} \mathrm{yr}$, we evaluate the critical eccentricity $e_{c r i t}$ associated with the boundary between chaotic and regular behaviour. The relatively infrequent case $e>e_{c r i t}$ leads to a short-lived chaotic interaction between the orbit and the tides. This implementation is based on a non-linear dissipation time scale and assumes total angular momentum conservation (see Mardling and Aarseth 1996 for details). The KS variables are then modified to the new energy and pericentre distance and corresponding polynomials are initialized during epochs of significant perturbation. Once this process terminates, or if $e<e_{c r i t}$ initially, the binary begins a slow periodic phase of circularization with small tides.

The treatment of tidal circularization employs a semi-analytical relation for the eccentricity: $e=f(q, n, a, t)$, with mass ratio $q$ and polytropic index $n$ (Mardling 1996). Here giants are modelled with an effective polytropic index which depends on the envelope mass (Mardling and Aarseth 1996). Angular momentum conservation yields the new pericentre $r_{p}=r_{p}^{0}\left(1+e_{0}\right) /(1+e)$ in terms of a previous pericentre distance and eccentricity. We adjust the associated $\mathrm{KS}$ variables to the new pericentre distance and velocity. If mass loss occurs or the motion is perturbed, the relevant parameters are re-initialized at pericentre. Except for the case of significant orbital expansion (or the formation of a compact object), the procedure terminates when $e=0$. Although the resulting shrinkage of the semi-major axis shortens the period, the condition for Roche mass transfer is usually not satisfied during circularization since, by angular momentum conservation, $a(1-e)$ actually increases.

\section{Mass Transfer and Orbital Decay}

Once a synchronous orbit has been achieved, Roche mass transfer may be initiated after further growth of the radius. The modelling of this process 
is based on a sophisticated scheme which distinguishes between the three relevant time scales (dynamical, thermal and nuclear) for the expansion rate of the stellar radius. The donor star is assumed to undergo Reimers-type mass loss in addition to a mass transfer rate of $1.0 \times 10^{4} \ln \left(R_{1} / R_{L}\right)^{3} m_{\odot} / y r$, where $R_{L}$ is the corresponding Roche radius (Tout 1990), and a certain fraction of this mass is accreted by the secondary. A rejuvenation procedure is carried out after significant mass change for main-sequence stars.

Common envelope evolution for giant or supergiant donor stars has also been included. This process may lead to a significant shrinkage of the semimajor axis, with physical collision in some cases. The subsequent evolution of short-period binaries $\left(a<3 R_{\odot}\right)$ may be subject to magnetic braking (Regös and Tout 1995) on a time scale $\tau_{m} \propto a^{5}$. If at least one binary component is a degenerate object, gravitational radiation may also lead to coalescence $\left(\tau_{g} \propto a^{4}\right)$ with an uncertain end result defined as a new type. We note that correction procedures are included for all dissipative processes in order to maintain an energy-conserving scheme.

\section{Recent Results}

The code development and testing of the various astrophysical processes is now essentially completed and permit a preliminary inspection of the results. Here we summarize some relevant features pertaining to the initial conditions described in Section 3. This model was studied until complete disruption, with a total life-time of $5.7 \times 10^{9} \mathrm{yr}$. Because of various timeconsuming astrophysical processes (e.g. Roche mass transfer), the actual CPU time was about $320 \mathrm{hr}$. By half-life $\left(t_{1 / 2} \simeq 1.9 \times 10^{9} \mathrm{yr}\right)$, there were $\simeq 1.1 \times 10^{9}$ individual integration steps and $\simeq 2.6 \times 10^{8} \mathrm{KS}$ steps. With some $3.8 \times 10^{7}$ block-steps, this corresponds to an average of about 30 particles per block-step and indicates an efficient usage of the eight pipes.

A simple summary is best carried out at the half-life $(N=5250)$. At this stage $N_{b}=290$, suggesting that the binaries are not preferentially depleted and therefore retain the capacity for preventing core collapse. The cluster half-mass radius expands from $r_{h}=0.8$ to 1.3 , during which some $1400 m_{\odot}$ are lost due to stellar evolution compared to a total initial mass of $6857 m_{\odot}$. Core collapse is only marginal when stellar evolution is included; the minimum core radius and membership noted was $r_{c} \simeq 0.06$ and $N_{c}=19$ at $t \simeq 2.2 \times 10^{9} \mathrm{yr}$. As the most massive single stars complete their evolution, the mass segregation of binaries becomes important. This is reflected in the enhanced abundance of binaries in the core, which increased to about 20 $\%$ by half-life. Hence it does not seem necessary to include a large initial binary fraction in order to halt core collapse and yield a significant observed abundance in the central regions. 
The behaviour of the binary distribution is of particular interest. On the dynamical side we note that 269 original binaries remain at $t_{1 / 2}$, with 20 exchanges and one new binary. Of the remaining population, there were 174 escaped binaries (one being a new binary and one a hierarchical triple); hence 36 binaries were either destroyed in dynamical interactions or suffered physical collision. The relatively small formation rate of binaries is noteworthy and can be understood as follows. During the process of hardening, a binary typically experiences $\simeq 10-20$ significant energy changes. Hence if only one of these interactions involve another hard binary, this can be sufficient to destroy the emerging binary, whereas in systems with no primordial binaries the probability of such encounters is much smaller. Interactions with binaries also produce some energetic or runaway escapers. Thus in the present model there where 54 dynamical ejections with terminal velocity $V_{f} / V_{t}>5$, six of which were binaries.

The astrophysics of close binary interaction in a dynamical environment presents many challenges. By the half-life stage some 24 binary orbits had circularized, of which five began as chaotic events. Following circularization, 13 binaries underwent Roche mass transfer with a high percentage reaching common envelope evolution. Of the 12 collisions, eight were defined as accretion-induced collapse (AIC), with one blue straggler and one star exceeding turn-off mass during Roche mass transfer. By the end of the calculation, there were 18 collisions, with 15 Roche cases, nine AIC, five blue stragglers and two Roche turn-off events. Finally, we note at least eight examples with both companions being white dwarfs, two of which had short periods $(\simeq 0.2$ and 0.6 days $)$.

\section{Future Prospects}

The attempt to synthesize stellar dynamics and stellar evolution represents a formidable challenge to the modern astronomer. Although the main ingredients are now in place, further refinements are required before observational comparison can be undertaken with confidence. In particular, we need a theory for Roche mass transfer for eccentric orbits and an improved treatment of giants for tidal circularization. Before making applications to globular clusters, we also need a synthetic stellar evolution scheme for population II stars and a convenient formulation for eccentric cluster orbits in a 3D galactic potential. Hopefully both of these features will be implemented in the near future. This will enable small $(N \simeq 25,000)$ globular clusters to be modelled on the more powerful HARP-3 (with 88 pipes) which has now been installed at Cambridge. However, open star clusters are also unique laboratories for studying various exotic objects and should not be overlooked. Of special interest here is the possibility of analysing 
such processes in detail, particularly the formation of blue stragglers and binaries containing degenerate components. Another important predicted cluster feature for comparison is concerned with the period-eccentricity relation which is now well established observationally (Duquennoy and Mayor 1991). Hence, we may say that a realistic star cluster simulation offers the opportunity of direct comparison with observations and in addition gives the theoretician a powerful tool for examining the interplay of complex processes involving both dynamics and astrophysics.

\section{Acknowledgements}

I thank my colleagues Drs. Peter Eggleton, Onno Pols and especially Chris Tout for advice on aspects of stellar evolution and Dr. Rosemary Mardling for implementing tidal circularization. Discussions with Dr. Jun Makino concerning the block-step scheme during several visits to Tokyo were also highly beneficial. This project has been supported by a cooperative scheme of the Royal Society, the Japanese Society for the Promotion of Science, and the British Council (Tokyo).

\section{References}

Aarseth, S.J. 1985, in Multiple Time Scales, ed. Brackbill, J.U. and Cohen, B.I. (Academic Press, New York), 377

Aarseth, S.J. 1992, in Binaries as Tracers of Stellar Formation, ed. Duquennoy, A. and Mayor, M. (Cambridge University Press), 6

Aarseth, S.J. 1994, in Galactic Dynamics and N-Body Simulations, ed. Contopoulos, G., Spyrou, N.K. and Vlahos, L. (Springer-Verlag), 277

Aarseth, S.J. 1996, in Binaries in Clusters, ed. Milone, E.F. (ASP series), in press

Duquennoy, A. and Mayor, M. 1991, Astron. Astrophys. 248, 485

Eggleton, P.P. and Kiseleva, L.G. 1995, Astrophys. J. in press

Eggleton, P.P., Fitchett, M.J., and Tout, C.A. 1989, Astrophys. J. 347, 998

Heggie, D.C. and Aarseth, S.J. 1992, Mon. Not. R. astr. Soc. 257, 513

Heggie, D.C. and Ramamani, N. 1992, Mon. Not. R. astr. Soc. 272, 317

Kroupa, P. 1995, Mon. Not. R. astr. Soc. in press

Kroupa, P., Tout, C.A. and Gilmore, G. 1993, Mon. Not. R. astr. Soc. 262, 545

Kustaanheimo, P. and Stiefel, E. 1965, J. Reine Angew. Math. 218, 204

Lyne, A.G. and Lorimer, D.R. 1994, Nature, 369, 127

Makino, J. 1991, Astrophys. J. 369, 200

Mardling, R.A. 1995, Astrophys. J. 450, 722

Mardling, R.A. 1996, in preparation

Mardling, R.A. and Aarseth, S.J. 1996, in preparation

McMillan, S.L.W., Hut, P. and Makino, J. 1990, Astrophys. J. 362, 522

McMillan, S.L.W., Hut, P. and Makino, J. 1991, Astrophys. J. 372, 111

Mikkola, S. and Aarseth, S.J. 1993, Celest. Mech. Dyn. Astron. 57, 439

Mikkola, S. and Aarseth, S.J. 1996, to be published

Regös, E. and Tout, C.A. 1995, Mon. Not. R. astr. Soc. in press

Spurzem, R. and Aarseth, S.J. 1996, to be published

Tout, C.A. 1990, Ph.D. thesis, University of Cambridge

Tout, C.A. 1995, personal communication 